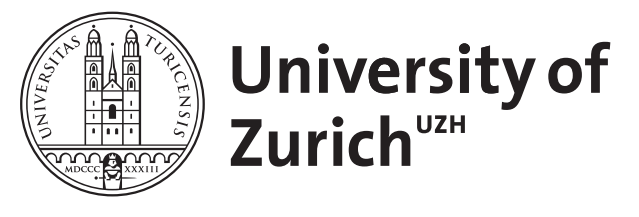

\title{
Im Gespräch mit Isidor Levin
}

\author{
Messerli, Alfred
}

\begin{abstract}
Anläßlich der Tagung Folklore als Tatsachenbericht (21.-23. September 2000), die von Jürgen Beyer und Reet Hiiemäe am Estnischen Literaturmuseum (Eesti Kirjandusmuuseumis) in Tartu organisiert worden war, hatte Alfred Messerli Gelegenheit, mit dem Erzählforscher und Folkloristen Isidor Levin zu sprechen. Von seinem Lehrer, Rudolf Schenda, wußte er von dem Gelehrten Isidor Levin als einer ,lebenden Legende‘. Teile dieses Gesprächs sind im folgenden wiedergegeben. Levin hat den transkribierten Text in Hinblick auf die Publikation in Fabula gelesen und mit Erweiterungen versehen
\end{abstract}

DOI: https://doi.org/10.1515/FABL.2009.016

Posted at the Zurich Open Repository and Archive, University of Zurich ZORA URL: https://doi.org/10.5167/uzh-26218

Journal Article

Published Version

Originally published at:

Messerli, Alfred (2009). Im Gespräch mit Isidor Levin. Fabula, 50(3-4):190-204.

DOI: https://doi.org/10.1515/FABL.2009.016 


\section{Im Gespräch mit Isidor Levin}

Anläßlich der Tagung Folklore als Tatsachenbericht (21.-23. September 2000), die von Jürgen Beyer und Reet Hiiemäe am Estnischen Literaturmuseum (Eesti Kirjandusmuuseumis) in Tartu organisiert worden war, hatte Alfred Messerli Gelegenheit, mit dem Erzählforscher und Folkloristen Isidor Levin zu sprechen. Von seinem Lehrer, Rudolf Schenda, wußte er von dem Gelehrten Isidor Levin als einer, lebenden Legende‘. Teile dieses Gesprächs sind im folgenden wiedergegeben. Levin hat den transkribierten Text in Hinblick auf die Publikation in Fabula gelesen und mit Erweiterungen versehen.

Messerli: Ich habe Ihren Aufsatz ${ }^{1}$ gelesen, und ich war sehr beeindruckt von der rigiden und klaren Haltung und dem theoretischen Ansatz, den Sie entwickeln. Ich würde aber gerne im Gespräch ein wenig herausfinden, wie Ihr biographischer Weg als Forscher verlaufen ist.

Levin: Bibliographie ist immer wichtiger als Biographie. Im hohen Alter könnte man viel zu einem behördlichen Curriculum angeben. Ich verzichte vorsichtshalber darauf. Nun werde ich nur darüber sprechen, was an und für sich für andere wissenswert sein dürfte.

Im Jahre 1947 war ich zum ersten Mal überhaupt in Rußland, da habe ich die Leningrader Universität besucht und wurde vor einem Gremium der Philologischen Fakultät vorstellig, um Examina für eine sowjetische ,Aspirantur“ in Folkloristik vor einer Kommission (deren Mitglieder Dekan Alexejev, der Ordinarius für russische Folkloristik Mark Azadovskij ${ }^{2}$ und der Märchenkundler Vladimir Propp $^{3}$ waren) abzulegen sowie nebenbei den sowjetrussischen Lehrbetrieb kennenzulernen. Da sich für mich in meiner Heimat angeblich keine Arbeitsstelle fand, gemäß meinem diplomierten Fach der Folkloristik, die ich noch bei Walter

1 Es handelt sich um einen unveröffentlichten Aufsatz in deutscher Sprache. Vgl. z.B. auch Levin, Isidor: Folkloristic Documentation, Textology and Editorial Principles: Methodological Paths from Fieldwork to Computer-aided Corpus. In: Storytelling in Contemporary Societies. Hgg. Lutz Röhrich/Sabine Wienker-Piepho. Tübingen 1990, 215-224.

2 Ders.: Mark Asadowski (1888-1954). In: Fabula 2 (1959) 176f.; ders.: Azadovskij, Mark Konstantinovič. In: Enzyklopädie des Märchens 1. Hgg. Kurt Ranke u.a. Berlin/New York 1977, 1114-1118.

3 Ders.: Vladimir Propp. An Evaluation on His Seventieth Birthday. In: Journal of the Folklore Institute 4,1 (1967) 32-49; ders.: A Belated Open Letter to Vladimir Jakovlevič Propp. In: Linguistica Biblica 23-24 (1973) 63-69; ders.: Der Märchenforscher Vladimir Propp. In: Märchenspiegel 3,1 (2001) 122-129.

Fabula 50 (2009) Heft 3/4

(C) Walter de Gruyter Berlin · New York

DOI 10.1515/FABL.2009.016 
Anderson $^{4}$ gelernt habe, geschweige denn in biblicis an der Theologischen Fakultät beim Professor der Bibelwissenschaften und Semitistik Uku Masing ${ }^{5}$, so wäre ich geneigt gewesen, an der Leningrader Universität zu arbeiten. Aber nach dem angeblichen Sieg über den ,Faschismus ‘ begann eine ähnliche Verfolgungswelle, und die Professoren selbst waren so zwischen Himmel und Erde. Die Zeit war für Intellektuelle, zumal für diejenigen nichtsowjetischer Herkunft, nicht günstig.

Aber immerhin habe ich 1949 einen Lehrauftrag bekommen, um in Leningrad Vorlesungen über estnische Sprache und Kultur am Lehrstuhl für finnischugrische Sprachen bei Dmitrij Bubrich ${ }^{6}$ zu halten. Ich habe damit begonnen, und es war, wie ich sagte, eine starke Verfolgungszeit. Bald hat man diesen Lehrstuhl von der Philologischen Fakultät verlegt; man hat eine Nordische Fakultät gebildet, und an dieser wurden die baltischen Sprachen abgeschafft. Die wollten weder mit Finnland noch mit Estland, das doch sowjetisch geworden war, Kontakte haben. Der Lehrstuhl wurde abgehackt, und das Baltische überhaupt ist weggefallen. Und da habe ich natürlich auch nichts mehr zu arbeiten gehabt. Der Ordinarius Bubrich hat mir vertraulich empfohlen, nach Tartu zurückzukehren, wo die ideologische Repression milder sein dürfte. Bubrich war dreimal verhaftet worden, infolge der Sprachpolitik in Karelien, das letzte Mal mit der Beschuldigung, daß er mit Wolfgang Steinitz ${ }^{7}$ verkehrte, ,dem bekannten deutschen Spion, der angeblich ugrische Sprachen bei Bubrich lernen wollte" (so die Anklage). Dabei war der Flüchtling und das Mitglied der KPD Steinitz zu jener Zeit schon in Schweden im Dienst der sowjetischen Botschaft. Als ich viele Jahre später Steinitz, jetzt Akademiepräsident der DDR, erzählte, was mir Bubrich gesagt hatte, war er empört und sehr dankbar für diese persönliche Information.

Hätte Bubrich noch sechs Monate gelebt, dann wäre er von Stalin, der Weisungen über Sprachwissenschaft abgab, bestimmt zum Wortführer der Linguistik gemacht worden, weil er stets den ,Marrismus ${ }^{\circ}$ ablehnte ...

Was war die Frage? Ja, Sie drängen aufs Biographische.

Messerli: Ja, Wissenschaftsgeschichte der akademischen Disziplin Folkloristik hat doch immer auch ihre biographischen Anteile.

Levin: Wenn Sie ein Zeitungsmann wären, ein Journalist, dann könnte ich das begreifen. Aber wenn Sie sich mit Wissenschaft befassen, sollte es doch auf argumentum ad hominem gar nicht ankommen ...

${ }^{4}$ Ders.: Walter Anderson $* 10.10 .1885$ in Minsk $-\nmid 23.8 .1962$ in Kiel. In: Deutsches Jahrbuch für Volkskunde 9 (1963) 291-311; Ranke, Kurt: Anderson, Walter. In: Enzyklopädie des Märchens 1. Hgg. ders. u.a. Berlin/New York 1977, 493f.

5 Levin, Isidor: Uku Masing (1909-1985). In: Fabula 27 (1986) 89-94; ders.: Masing, Uku. In: Enzyklopädie des Märchens 9. Hgg. Rolf Wilhelm Brednich u.a. Berlin/New York 1999, 387f.

6 Dmitrij Vladimirovič Bubrich (1890-1949), russischer Finnougrist, seit 1925 Inhaber des Lehrstuhls für finnisch-ugrische Sprachen an der Universität Leningrad.

7 Schellbach-Kopra, Ingrid: Steinitz, Wolfgang. In: Enzyklopädie des Märchens 12. Hgg. Rolf Wilhelm Brednich u.a. Berlin/New York 2007, 1227-1230.

8 Nach dem russischen Linguisten Nikolaj Jakovlevič Marr (1865-1934), dessen weithin rezipierte Theorie zur Sprachentwicklung durch Stalins „Sprachwissenschaftliche Briefe" ihre Geltung verlor. 
Messerli: Das ist natürlich eine Annahme von Ihnen. Selbstverständlich kommt es auf die Person an.

Levin: Leider! Also, ich möchte meine Geschichte fortsetzen. Es wurde dann die ganze Universität Leningrad als ideologisch dubios umgekrempelt. Es begann eine Aktion gegen ,Kosmopolitismus' (früher als marxistischer ,Internationalismus ' und dergleichen gelobt). Ich war noch ziemlich jung, hatte zwar den Dorpater Magistertitel, aber sonst keine besonderen Verdienste. Im Gegenteil, ich war suspekt, weil ich unter nazi-deutscher Besatzung das KZ überlebt hatte. Als Märchenkundler war ich bereits ein Kosmopolit, und deswegen mußte ich auf der Hut sein und allerlei Diskriminierung ertragen.

Nach diesem Zwischenfall, wenige Monate danach, ist der Leiter des Lehrstuhls, Bubrich, gestorben - Infarkt. Und meine Verbindung zur Universität in Leningrad hat sich damals automatisch aufgelöst. Ich kehrte nach Estland zurück, als ,Privatgelehrter', den es in der Sowjetunion überhaupt nicht geben durfte. ,Arbeitsscheue' bzw. arbeitlose Personen durfte es nicht geben. Für meine sowjetisierte Alma mater war ich ein Stiefsohn.

Für Sowjet-Estland waren meine Schule, meine Richtung, meine Lehrer, alles war sozusagen suspekt. Walter Anderson hatte das Land verlassen, er wollte, wie er mir beim Abschied 1939 sagte, nicht die sowjetische Herrschaft noch einmal erleben. Mein Lehrer in judaicis war Professor Lazar Gulkowitsch'. Sein Lehrstuhl wurde gleich 1940 beim Anschluß Estlands an die UdSSR liquidiert, er blieb erwerbslos und wurde 1941 unter deutscher Besatzung samt Familie mit den Juden ermordet. Die Theologische Fakultät, wo Professor Uku Masing lehrte, bei dem ich Bibelwissenschaft und Semitistik studiert hatte, wurde von den sowjetischen Besatzern geschlossen, denn, so hieß es, weil es keinen Gott gibt, braucht man keine Religionswissenschaftliche Fakultät. Daß es in Leningrad an der Akademie ein Institut für Orientalistik gab, wo die Bibelsprache erforscht wurde, war für die einheimischen Machthaber in Estland nicht maßgebend. Wir blieben erwerbslos. Es war eine schwere Zeit.

Professor Vladimir Propp beispielsweise, der für folkloristische Komparatistik zuständig war, wurde gerügt. Wissen Sie, warum? In seiner Dissertation über Märchen hatte er zu viele Ausländer zitiert. Und das wurde ihm von allerhöchster Stelle aus verübelt. Stalins Kreatur Ždanov und die Partei haben sich in die schmutzige ,Säuberungsaktion' eingeschaltet. Kurz und bündig: Es hat nicht viel gefehlt, daß er zum zweiten Mal verhaftet worden wäre, zumal Propp als Deutscher in Leningrad überhaupt nicht mehr wohnen durfte.

Azadowskij, der erste führende Mann, der einen Lehrstuhl für russische Literatur innehatte, wurde auf der Leningrader Konferenz gegen Kosmopoliten allerhöchst von Ždanov bezichtigt, daß er das russische Volk verleumdet hätte, indem er nachgewiesen hatte, daß das beliebte russische Märchen vom Fischer und seiner Frau aus Grimms Kinder- und Hausmärchen, und zwar in französischer Übersetzung, entlehnt und lediglich von dem beliebtesten russischen Dichter Puškin in

9 Lazar Gulkowitsch (1898-1941), aus Weißrußland gebürtiger Judaist, der 1924-33 in Leipzig, 1934-41 in Dorpat lehrte. 
Verse gesetzt worden war ... Das galt fast als Hochverrat. Azadovskij wurde kurzerhand aus dem Dienst an der Universität entlassen und seiner Stelle als Leiter der Abteilung Folklore an der Akademie der Wissenschaften enthoben, und das ausländische Wort Folklore wurde patriotisch durch ,Volksdichtung ' ersetzt.

$\mathrm{Zu}$ jener gefährlichen Zeit hatte noch kein Professor eine Rente bekommen. Ein Professor wirkte, so lange er auf den Beinen war (wenn er verhaftet wurde, dann war das etwas anderes). Aber diesmal, im Fall Azadovskij, hat man einen Präzedenzfall statuiert. Man hat einen Menschen entlassen, ihm das Recht, öffentlich Vorlesungen zu halten, inoffiziell, aber wirksam entzogen. Er war genötigt, Bücher seiner Privatbibliothek fürs tägliche Brot zu verkaufen. Dennoch war es ihm vergönnt, in seinem Bette zu sterben. Das war eine Gnade. Üblicherweise wäre er verhaftet und in seine sibirische Heimat deportiert worden.

Nach einem Jahrzehnt, als Stalin schon gestorben war, kam ich noch einmal nach Leningrad. Ich war der erste, der einen Lehrauftrag von Professor Viktor Žirmunskij ${ }^{10}$ erhielt, freilich auf Verfügung des Generals Tjulpanov ${ }^{11}$, der gerade stellvertretender Rektor der Universität Leningrad geworden war und mir in dieser zivilen Eigenschaft eine Audienz gewährte. Ich habe zum ersten Male an dieser Universität einen Lehrgang für deutsche Volkskunde etabliert (in deutscher Sprache vorgetragen, sonst mußten sämtliche Fächer in russischer Sprache gelehrt werden). Das war eine liberale Zeit, und General Tjulpanov, der in der sowjetischen Besatzungszone in Berlin Chef der Informationsverwaltung war, war im Grunde ein Freidenker. Aber dieser Liberalismus dauerte nur bis zu den Ereignissen in Ungarn. Dann wurde mir meine Lehrbefugnis nicht mehr verlängert, d.h. wieder entzogen. Das war nun schon zum zweiten Mal. Mir wurde vorgeworfen, daß ich in der westdeutschen Zeitschrift Fabula eine , antisowjetische "Besprechung (NB! ohne Zensur) über ein Buch udmurtischer Märchen publiziert hatte ${ }^{12}$. Es hospitierte in meiner Vorlesung ein Offizier der ersten Abteilung (d.h. KGB), der Deutsch verstand. Er kritisierte erstens, daß ich das Wort ,Deutschland“ gebrauche, wo es dieses Land doch nicht mehr gäbe. Ich erwiderte, daß in OstBerlin eine Zeitung unter dem Titel Neues Deutschland täglich erscheine. Ungut war auch, daß ich über Volksbräuche und Sitten im ,bürgerlichen ' Westdeutschland, aber weniger oder kaum über die neuen Bräuche und die proletarische Volkskultur in der fortschrittlichen DDR redete. Ich hatte Zulauf von Studenten, zur Unzufriedenheit anderer Dozenten, die Lehrveranstaltungen angeboten hatten. Aber, wie gesagt, ich war Kummer gewohnt.

Ich habe Ihnen flüchtig angedeutet, daß ich inzwischen privatim eine Methodik für Forschungen auf dem Gebiete der Folkloristik entwickelte. Da wurde ich ab 1967 nacheinander an zwei Institute, und zwar nicht in Rußland, sondern in Asien berufen. Es ging darum, eine ,Dokumentationsstätte' (nicht ,Archiv', das grund-

${ }^{10}$ Viktor Maksimovič Žirmunskij (1891-1971), russischer Germanist.

${ }^{11}$ Sergej Ivanovič Tjulpanov (1901-84), sowjetischer Kulturpolitiker, Leiter der Zensurund Propagandaabteilung der Militärverwaltung der sowjetisch besetzten Zone, danach Professor in Leningrad.

12 Levin, Isidor: Die Volkserzählungen der Wotjaken (Udmurten) (Mit Beiträgen von Walter Anderson). In: Fabula 5 (1962) 100-155. 
sätzlich den Vorschriften des KGB unterstellt sein mußte) zu gründen und dabei verschiedene Methoden zu etablieren. Die Hauptsache war, örtliche nationale Kader heranzuschulen. Unter diesem Vorwand durfte ich in Leningrad wohnhaft bleiben, aber für die Akademie in Tadschikistan am Pamir und dann auch gleichzeitig im Kaukasus, in Armenien, offiziell arbeiten. Das war eine einmalige Situation. Und mir wurde es möglich, meine extremsten Ideen in der Folkloristik zu verwirklichen. All das hatte in der Sowjetunion nichts Vergleichbares. Auf verschiedenen Tagungen und Kolloquien, wo ich schon früher willkommen war, weil ich doch ein sowjetisches Hochschuldiplom in russischer Sprache und Literatur, eine Aspirantur in diesem Fach sowie noch eine beim Institut für Orientalistik nachweisen konnte, hatte ich meine Ansichten darüber geäußert, was zu tun und was dringend zu lassen wäre.

Die Forscher in der Sowjetunion aber, besonders in Leningrad und Moskau, waren dürftig gebildet und schon darum ideologisch erzkonservativ. Sie waren, wie fast alle Parteigenossen und Funktionäre im akademischen Betrieb, erfahrene ,Konjunkturreiter'. Die wollten immer mit der ,Parteilinie " konform sein. Wenn die Partei und Brežnev etwas verkündigt hatten, wollte man das sofort realisieren. Z.B. befaßten sich mehrere Institute mit dem Entwurf von ,Traditionen' und Bräuchen fürs Volk, die Brežnev gern hatte. Man steckte tief im romantischen Ethnismus, also: Soundso viele Völker hat Gott bzw. die Natur geschaffen, und jedes Volk wurde mit unvergleichlichen, also höchst positiven Tugenden versehen, welche die Wissenschaftler nachweisen und auslegen sollten. Und in diesem Rahmen mußte man denken, arbeiten und forschen. Was kann man dabei an Wissen schon erzielen?

Messerli: Sie hatten den Auftrag, die Folklore Tadschikistans zu erfassen. Sie bekamen ein Team dafür. Wann hat das angefangen, und wie sah konkret die Arbeit der Leute, die Sie ja schulen mußten, aus?

Levin: Es hat damit begonnen, daß zwei Mitarbeiter vom Akademieinstitut in Tadschikistan nach Leningrad kamen mit dem Typoskript eines Buches tadschikischer Märchen, und sie wollten ein Gutachten der Folkloristen des PuškinInstituts der Akademie bekommen. Es wurde eine Sitzung einberufen, ich war als bekannter Märchenkundler und Orientalist eingeladen. Ich habe mit Kritik nicht gespart, suchte es ihnen zu begründen und erklärte, wie man ein Märchenbuch vorbereiten und dann erst herausgeben könnte. Die Gäste baten mich, sie privat zu empfangen. Das geschah, dabei zeigte ich ihnen die jüngsten Märcheneditionen. Die Gäste fuhren nach Hause, sichtlich beeindruckt. Bald erhielt ich vom Direktor ein Telegramm mit einer Einladung, für Konsultationen zu kommen. Dann besuchte mich der Vizedirektor und bemühte sich, mich dafür zu gewinnen, die Folkloristen zu beraten. Ich flog da hin (in einem neunstündigen Flug) und erklärte mich bereit, ein Projekt zu unterbreiten. Kurz: Ich wurde sofort berufen, die Arbeit der Abteilung Folklore wissenschaftlich zu leiten.

Den nationalen Republiken hatte man sehr großzügig volkstümliche Fächer finanziert, damit man sich sozusagen auch im Ausland zeigen konnte. Und die Universität hatte immer mehr Abgänger. Jahrein, jahraus kamen neue Kader. Jeder mußte eine Arbeit finden. Beim Abgang mit dem Diplom bekamen sie eine 
Anweisung, sie sollten sich an dieser und jener Stelle melden, zwecks Arbeit. Sie konnten das nicht selbständig auswählen. Also wohin? Entweder wurden sie Lehrer an einer Grundschule oder sie arbeiteten in einem akademischen Institut.

Eines dieser Institute in Duschanbé hieß ,Institut für Sprache und Literatur', und in Tadschikistan hatte man eine Abteilung für Folklore - ein Fach, das jeder zu kennen glaubte. An die 35 Personen waren dort angestellt. Das war noch vor meiner Zeit. Man wußte nicht, wie man die Leute beschäftigen konnte. Was sollten sie tun?

Bei meinem ersten Aufenthalt 1966 in Tadschikistan habe ich einige Vorlesungen über Märchenforschung gehalten. Über die Geschichte und die Probleme dieses Faches wußten sie nichts. Ich habe ihnen mein Projekt vorgestellt: „Hier sehen Sie, da sind kahle Wände, nackte Wände. Wenn Sie jetzt beginnen, dann werden Sie zu einer bestimmten Zeit hier Karteien sehen. Die Karteien werden Ihnen das und das zeigen. Machen muß man das auf eine bestimmte Weise, die ich Ihnen zeigen werde." Um das experimentell nachzuweisen, habe ich folgendes getan: Die lieben sehr ihre Vierzeiler. Ich nahm einen Text mit vier Zeilen und fragte: „Können Sie mir sagen, ob Sie von diesem Text irgendwelche Belege haben?“ „Ja, selbstverständlich“, sagte einer. „Ich habe das selbst schon aufgezeichnet.“ „Dann sagen Sie mir, wo liegt das jetzt? Wie kann ich es zu sehen bekommen?“ Er hat es gesucht und nicht gefunden. Da habe ich gesagt: „Das ist es eben. Normalerweise sollte man diesen Vierzeiler innerhalb von zwei Minuten ausfindig machen können, wenn alles richtig durchgearbeitet ist." Und so habe ich sie überzeugt, daß sie nach Instruktionen arbeiten sollten. Die Aufgabe wurde in ,Operationen', in einzelne Prozeßschritte, zerlegt und chronometriert. Denn was war die Arbeit an einem sowjetischen akademischen Institut sonst? Man mußte Aufsätze schreiben, die keiner brauchte. Dann saßen sie für etwa 22 Seiten Text (einen Druckbogen) ein halbes Jahr, und sie verfertigten dann nach russischem Muster, mit eigenem Material in der Muttersprache, ein Schriftstück. Aber das war doch keine Wissenschaft.

Also, ich hatte dort Einfluß gewonnen, habe der Obrigkeit erklärt, was man mit meinem eigens für Tadschikistan entwickelten System (TSL) erreichen würde, wie das anzustellen und wozu es gut sei. Das Verfassen , wissenschaftlicher Arbeiten" habe ich in meinem Sektor abgeschafft. Das war gewagt, man klagte bei der Parteiorganisation, ich sei ein Schädling. Ich habe jede Aufgabe, die Arbeit für jeden Teilnehmer, programmiert und chronometriert. Denn in der Sowjetunion war es Pflicht für jeden, acht Stunden pro Tag zu arbeiten. Ich habe mit sechs Stunden gerechnet. Es hat sich bald herausgestellt, daß die meisten nur vier Stunden pro Tag gewillt waren, etwas zu tun. Entsprechend hat sich die Erfüllung des offiziell bestätigten Plans verzögert. Wenn ich anwesend war, dann wurde unter meiner Aufsicht und mit Erklärungen wie im Seminar noch fleißig gearbeitet. Wem es nicht paßte, der wurde bald entlassen, er wurde dann anderswo angestellt. Ich habe ihnen versprochen, daß sie planmäßig unter meiner Leitung Dissertationen schaffen würden als Teil kollektiver Arbeit. Das hat angespornt, weil von einem akademischen Titel ihr Gehalt abhing. Nachdem ich wegen der Hitze nach Leningrad flog, hat man der Belegschaft zusätzlich Urlaub gewährt, denn sie 
waren müde. Oder man fuhr in die Heimat, für folkloristische ,Feldarbeit', oder um bei der Baumwollernte zu helfen. Dafür wurden auch die Schulen eingesetzt.

Zuerst wollten wir die Grundlage, eine geordnete Quellenbasis, schaffen. Es gab viele Belege, etwa rund eine halbe Million Texte. Um das Nötige problembezogen finden und bearbeiten zu können, mußte man alles, Schritt für Schritt, planmäßig bearbeitet haben.

Messerli: Sie hatten also schon von Anfang an dieses klare Konzept?

Levin: Absolut. Ich habe alle Züge, alle Schritte theoretisch und praktisch auf solider Basis ausgearbeitet. Ich hatte also 35 Personen, Frauen, aber auch Männer, zur Verfügung. Es war im Jahr 1966. Ich habe da erstens Vorlesungen zur Einführung in die Folkloristik gehalten; zweitens habe ich Arbeitsanleitungen in Gestalt von Instruktionen gegeben; und jeder wußte, was er zu tun hatte und wieviel er zu leisten verpflichtet war. Am Fließband haben wir da kollektiv gearbeitet. Und am Ende bildeten sich Karteien. Zuerst ging es um die analytische Erfassung des gesamten Materials.

Messerli: War das Material schon da oder mußte es in Feldforschung erst gewonnen werden?

Levin: Feldforschung fand auch statt. Mengen von Stoffen wurden schon früher aufgezeichnet. Die Sammlung war aber gänzlich in Unordnung. Und keiner wußte, wo etwas war. Das erste, was zu tun war, war das Paginieren der Manuskripte. Man hat alle Papiere mit Stempel durchpaginiert. Und nun war es möglich, auf die erste Frage A (Wo im Archiv?) zu antworten. Die Angabe lautete: Es befindet sich auf Seite soundso im Teil soundso. Ein ,Teil‘ enthielt jeweils 9999 Seiten. Die Teile wiederum bildeten eine ,Serie', bezeichnet mit zwei Buchstaben und zwei Ziffern. Alles war numeriert. Das war eine Neuheit. Das hatten auch die Russen nicht. Bei diesen hat jemand vor 150 Jahren die wenigen Schränke per $\mathrm{ABC}$ numeriert. Die Letten hatten eine Zählung pro Text. Die Zahlen gingen in die Millionen.

Dann kommt die Frage zwei mit dem Index B: Wo hat man den Text aufgezeichnet? Nun, man kann natürlich schreiben, im Bezirk soundso, im Land soundso, oder sogar in einem Dorf. Man wußte aber nicht, wo es liegt - das taugte also nicht. Die Welt, die Landkarte, ist bekanntlich in Trapeze eingeteilt, die man mit zwei Ziffern und einem Buchstaben bezeichnet. Aber ein Trapez ist eine zu große Fläche, da teile man sie in $3 \times 3=9$ Teile. Und jedes Neuntel teile man in $4 \times 4$ Quadrate. Falls nötig, teile man das Quadrat in vier Teile (a, b, c, d). Damit die Mitarbeiter verstehen konnten, worum es bei der Lokalisierung ging, habe ich von einem, der zeichnen konnte, eine Karte von Tadschikistan anfertigen lassen mit allen erwähnten Koordinaten. Die Landkarte gleich von Anfang an in kleine Quadrate einzuteilen, taugt nicht, denn im Manuskript werden nur selten genaue Ortsdaten angegeben. So konnte man präzise in der zweiten Zeile mit einem kurzen Kode von acht Stellen den Aufzeichnungsort für jeden bestimmten Text angeben. Das Toponym wird notiert, wie es im Text steht, das ist für Historiker wertvoll. So bekommt man automatisch ein Kartogramm von der Verbreitung eines Phänomens im Raum. Das läßt sich sinnvoll interpretieren. 
Nun folgt die dritte Zeile, Index C, mit der Frage: Wer hat dem Archiv den Text geliefert? Die Person des Vermittlers wird mit Familien- und Vornamen sowie Geschlecht (m/f), ferner mit der Altersgruppe (von 1 bis 9) notiert. Die vierte Zeile enthält die Frage: Aus wessen Mund wurde der Text aufgezeichnet? Meistens ist nur der Vorname bekannt, sonst schreibe man die Angaben wie in der dritten Zeile. Falls der Erzähler nicht vom Aufzeichnungsort stammt, muß man seine Heimat wie in der zweiten Frage angeben. Die fünfte Frage ist: Wann wurde der Text aufgezeichnet? Die Antwort mit Index E braucht acht Ziffern: für die Jahreszahl vier, für den Monat zwei und den Tag auch zwei. Was unbekannt ist, schreibe man als X, nicht als 0. Benutzt wurde der europäische Kalender. Die sechste Frage ist für die Registrierung sehr wichtig, sie lautet: Was ist der Inhalt des Textes?

Messerli: In dem Papier, in dem Sie ihre Methode darlegen ${ }^{13}$, zeigt sich auch eine Skepsis gegenüber den historischen Grenzen. Hängt das mit Ihrer Biographie zusammen?

Levin: Grenzen hängen von administrativ-politischen Umständen ab.

Messerli: Und Sie sind ja an sehr großen Räumen interessiert ...

Levin: Ja. Ich gehöre der Andersonschen geographisch-historischen Schule in der Volkskunde an. Mir kommt es auf Kartogramme an.

Messerli: ... und zielen deshalb auf eine eindeutige Angabe, die nicht von politischen Wechselfällen abhängt.

Levin: Genau. Orte oder Gegenden wechselten sehr oft den Namen. Damit darf man sich nicht binden. Man muß unveränderbare Koordinaten haben. Und das Ziel war, Kartogramme zu bekommen. Ganz gleich, wovon. Denn Kartogramme zeigen ganz bestimmte Grenzen einer Verteilung im Raum. Diese Grenzen sind kulturhistorisch relevant. Auch wenn Sie kartieren, etwa wo man mehr Seife kauft. Die Verwendung von Seife ist wahrscheinlich ein Indiz für kulturelle hygienische Sachverhalte. Es kommt mir nicht auf Seife oder andere Konsumgüter an, sondern auf die fragwürdige Tatsache einer Grenze, einer lokalen, folglich auch historischen Zugehörigkeit, die Bindung einer Bevölkerung. Wenn ich nun solch ein Kartogramm habe, und wenn ich noch zwanzig oder zweihundert oder mehr solcher Kartogramme habe, ganz gleich, wovon, dann sehe ich, daß hier irgendwelche Grenzen zum Vorschein kommen. Dann frage ich mich, was ist das für ein Territorium? Wie, in welchem Sinne, zeichnet es sich vor anderen Territorien aus? Und das läuft auf Historiographie hinaus. Man schafft ein neues volkskundliches Instrument, es ist ein regionaler, auch globaler Kulturatlas. Zum Beispiel: Ich sehe, es wurde da gesammelt. Aber wenn ich jetzt schaue, wieviel da in einem Jahr und dann im darauffolgenden Jahr und so weiter gesammelt wurde, dann frage ich mich: Warum? Warum wuchs es anfangs an und hat dann plötzlich aufgehört? Was steckt dahinter? Es kann etwas banal Materielles sein, zum Beispiel, daß man kein Geld hatte, Leute hinzuschicken. Aber dahinter kann sich auch eine gesellschaftlich relevante Erscheinung verbergen. Oder ich kann herausfinden: Wie ist die Struktur der Generationen in unserem Material? Und wenn

\footnotetext{
${ }^{13} \mathrm{Vgl}$. Anm. 1.
} 
ich merke, daß die Leute nur immer von älteren Menschen Aufzeichnungen machen, dann steckt dahinter eine Theorie, daß die alten Leute alles besser wissen, was gewesen ist, sie dem ,Ursprung des Volkes“ näher seien. Und was später kam, sei nicht mehr authentisch. Einerseits kann es stimmen, aber andererseits ist es eine voreingenommene romantische Konzeption. Oder ich sehe zum Beispiel, daß man im Jahre soundso oder im Jahrzehnt soundso nur Männer befragt hat, als ob überhaupt keine Frauen da wären. Ist die Bevölkerung nur männlich gewesen? Nein. Aber die Sammler waren Männer, und ein Mann im Orient hat nicht die Möglichkeit, eine Frau zu befragen. Aber als man die Frauen, die von der Universität kamen, hinschickte, hat sich das geändert. Also, solche Erscheinungen können leicht erklärt werden, und das hat mit Soziologie zu tun.

Nebst Topographie und Chronologie, also Verteilung in Raum und Zeit, ist die $\mathrm{S}$ c h i c h t u n g von Erscheinungen in der Gesellschaft für die Volkskunde maßgebend. Man lerne mit Kartogrammen, Chronosoziogrammen zu arbeiten; sie lassen sich auch quantitativ sinnvoll korrelieren.

Messerli: Kehren wir zur Systematik zurück.

Levin: Die schwerste, die sechste Frage der Klassifikation (Index H) lautet: Was wurde aufgezeichnet? Das läßt sich schnell anhand eines Kodifikators mit einem Buchstaben für die H-Zeile hinreichend bezeichnen. Ich vermied jede Terminologie und versuchte, die Welt der Volkskunde einleuchtend zu gliedern und zu kodieren.

Messerli: Haben Sie nach Prosa und gereimten Formen unterschieden?

Levin: Ja. Es war aber wichtig, daß auch ungebildete Mitarbeiter Dinge unterscheiden konnten, wenn sie die Listen des Kodifikators aufmerksam gelesen hatten. Die Welt der Volkskunde zu überblicken in ihrer Gänze, ist nicht wenig. Es ist wie mit einer Menükarte oder in einem Warenladen. Man wählt sich aus dem Angebot das, was einem paßt. Ein Gelehrter wird solche Listen an und für sich zu schätzen wissen. Darin steckt Wissen über Leben und Methode. Ich hatte es schwer, einen Kodifikator zu erstellen, damit es die Kollegen leicht hatten.

Messerli: Haben Sie etwa auch darauf hingewiesen, ob der Erzähler in der Erzählung vorkommt und dergleichen? Ob es sich um eine autobiographische Erzählung handelt oder es mehr um formelhafte Dichtung geht?

Levin: Sie dürfen nicht vergessen: Hier geht es um die Registrierung, um die Erfassung des Materials. Sie können hier nicht gleich in die Tiefe gehen; Sie müssen auf einem bestimmten Niveau notieren. Auf die sechste Frage (Was?) muß man eine klare, eindeutige Antwort geben, die ohne Problem mit Leuten, die wenig kosten, realisiert werden kann. Da kommen zwar die ,Gattungen ' vor, sie müssen aber mundgerecht gemacht werden. Denn was in einer Forschungsstelle so heißt, heißt bei einer anderen Forschungsstelle anders. Und die mundartlichen Bezeichnungen kommen auch ins Spiel. Also, eine gelehrte Nomenklatur taugt nicht. Das ist in Estland geschehen. Und als ich erkannt habe, daß es ein Fehler war, habe ich diesen Fehler nicht mehr gemacht.

Viele haben eine ganze Reihe von ,Gattungen“ oder ,Textsorten“ mit einem Kürzel versehen, das ist hübsch anzusehen. Es gab von den Kürzeln etwa hundert Stück. Die Kürzel wurden nach dem ABC sortiert. Aber nach dem ABC etwas zu 
ordnen, ergibt keine sachliche Ordnung, sondern eine erhabene Unordnung. Und das hat sich schließlich fast überall gezeigt. Außerdem sind neue Terminologien hinzugekommen ... Finessen in Gattungstheorien sind meines Erachtens von Übel, das habe ich von Anderson gelernt. Man muß also ganz einfache, idiotensichere Gruppen bilden können, und dann wird es funktionieren. Nehmen wir zum Beispiel Prosa: Erzählt der Mann von sich oder von anderen? Ich sage nicht: Das ist eine Sage oder das ist keine Sage, sondern ein ,Memorat'. Ich gebe das an, was man erkennen kann: Es ist ein Märchen.

Messerli: Woran erkennen Sie das Märchen?

Levin: Ich nenne es nicht Märchen, aber es läßt sich erkennen. Es ist ein bestimmter Inhalt, und es gibt am Anfang und am Schluß bestimmte Formeln. Ob es ein Märchen oder eine Sage ist, ist mir vorerst völlig gleich. Beide Erzählungsarten haben bestimmte ,Motive' oder nicht. Um zu sagen, daß es sich um Prosa handelt, genügt ein Kode. Was wurde inhaltlich aufgenommen? Um darauf zu antworten, ist eben der Kodifikator da. Und damit Inhalte in dreistelligen Ziffern nicht verwechselt werden, gibt es für jede Frage der Klassifikation und Qualifikation einen Index als Buchstaben.

Da kommen Sie auf H8, das wird Prosa sein, H9 werden Lieder sein. Hier habe ich vorläufig nur zwei Stellen für Ziffern vorgesehen. Denn wir möchten auch wissen, wovon erzählt oder gesungen wurde. Da muß man wohl für den Inhalt etwa der Sagen einige Positionen aus der Sparte H6 (Wissen, Glauben und Verhalten) leihen, damit alle Texte zugehörigen Inhalts, ungeachtet der Gattung, zusammen abgerufen werden. Mit einer Kombination von einem Buchstaben $\mathrm{H}$ und drei Ziffern, also vier Stellen, läßt sich eine primäre inhaltliche Information darüber, was erzählt, gesprochen oder gesungen wurde, geben. Der Kodifikator beschreibt auch strukturell, formell Spiele und Gesang, mit oder ohne Worte, auch reine Musik. Das alles läßt sich interpretieren. Auch das Wissen läßt sich dreistellig kodieren.

Messerli: Ich habe noch nicht verstanden, wie Sie das machen.

Levin: Nehmen wir zum Beispiel eine Erzählung - etwas, das Sie Sage über Ereignisse in Natur oder Gesellschaft nennen würden, etwa über heilige Orte oder über heilige Zeiten oder dergleichen. All das läßt sich sehr kurz machen. Ähnlich, wenn es sich um Volksmedizin handelt oder Volksphysik, Volksastronomie. Alles, was wir in der , hohen ' Schul-Wissenschaft haben, gibt es auch in der Folklore als Aberwissen, d.h. ,gesunkenes Kulturgut' oder vulgo ,Aberglauben'. Auch das läßt sich ganz einfach kodieren.

In Armenien (ASL) wurden die Texte mit dem Computer registriert, in Tadschikistan (TSL; diese Kürzel stammen von Archer Taylor, der sie in unserer Korrespondenz gebrauchte) noch mit Tabulator (Lochkarten); das hatte aber keinerlei Einfluß auf mein System. Es läßt sich auch mit Bleistift oder mit Feder und Tinte realisieren. Texte werden ja nicht klüger, wenn man sie digitalisiert. Weil die Folkloristik eigentlich d i e Wissenschaft von der Varianz eines Stoffes ist, der infolge der Überlieferung entsteht und fixiert worden ist, muß alles diesem Ziel der Erfassung der Varianz auf Typenebene unterstellt sein. Ich würde eine Formel gebrauchen, die Hitler benutzt hat und welche die BBC ironisierte: „Ich habe alle 
Möglichkeiten von vornherein einkalkuliert.“ Er hat sich glücklicherweise für die Menschheit verrechnet. Ich habe alle empirischen Möglichkeiten des Irrtums als erfahrener Pädagoge einkalkuliert. Wenn es etwa ganz unmöglich ist, im Kodifikator eine Stelle zu finden, dann gehört das zur nächsten Gruppe 0.

Messerli: Welche Sachgebiete haben Sie vorgesehen?

Levin: Zuerst gemäß Zeile H ist es das Haus, das Wohnen im Raum (H1). Nachrichten über solche Dinge lassen sich einfach kodieren. Dann kommt unter H2 der Mensch und sein Äußeres. Wie mache ich das? Mittels eines Schemas des Menschen von Kopf bis Fuß, das ich grob einteile. Und wenn Sie sagen wollen, zum Beispiel, er trägt einen Ring, dann muß man zuerst einen Kode für Arm und Finger haben. Das ist einfach. Nun braucht man eine Sparte für ,Kleidung ' und Attribute bzw. für Schmuck. Wenn aber zum Beispiel von Krankheiten der Hand, der Finger die Rede ist, dann muß es eine Möglichkeit geben, ,Entstellungen ' zu registrieren, etwa der Hand bzw. des Fußes, des Auges oder des Ohres, wo auch über Lahme und Blinde, also Behinderte, erzählt wird. Das läßt sich einfach machen, indem man eine Sparte für ,Krankheiten ' beliebiger Körperteile in der ,Was-Zeile' bei H2 vorsieht. Und für jeden Fall brauche ich genau drei Ziffern. Auch untere oder obere Kleidung gehört dazu. Beim ,Kopf' kommen als ,Kleidung 'Hüte oder Mützen vor; für die Füße sind es Schuhe, Socken, die Sie mit H2 kodieren. Bei solcher Systematik werden verschiedene ,Kleidungen“ von sämtlichen Körperteilen zusammenkommen, und Sie erhalten sofort ein Kartogramm etwa von den Mützen oder Schuhen, und deren Varianz kommt in extenso aus allen Gattungen, z.B. der Witze oder Lieder, also konkrete Belege für Kleidungsstücke, die für Magisterarbeiten ausreichen sollten. Das ist das Endziel der Dokumentation nach dem ,System Levin “ für Iranisch-Tadschikisch sowie Armenisch, letztens auch global Jiddisch (was das Wissenschaftskolleg zu Berlin haben wollte). Es wäre nützlich, wenn weitere Dokumentationsstätten für Volkskultur ihr Material in dieser Weise einheitlich ordneten.

Messerli: Welche Sparten außer ,Mensch' gibt es weiter?

Levin: Arbeit (H4), Hobby (H5), Sprachgutformeln, einschließlich Sprichwörter und Redensarten, Formeln für Begrüßung und Abschied, Fluch- und Segensformeln, Formeln, mit denen man zum Trinken nötigt und so weiter (H7). Die grundsätzliche Instruktion lautet: Auf die ersten sechs Fragen darf man nur eine eindeutige Antwort geben. Nur einen Kode, nicht zwei. Dann werden alle Varianten in der Synopse eines ,Typs ' sichtbar mechanisch zusammenkommen. Das nenne ich die primäre Klassifikation. Anschließend müssen wir noch in vier Gruppen von Informationen kodieren. Das nennen wir Qualifikation. Dafür können Sie mehrere dreistellige Ensembles von Ziffernkodes verwenden. Eines neben dem anderen.

Also, wie funktioniert das? Wir sind doch bekanntlich am sozialen Leben interessiert. Da muß man eine Liste Index K auf dem Kodifikator haben, in der die Bevölkerung, die Gesellschaft in soziale Gruppen gegliedert und kodiert wird. Erstens ist hier die Altersgruppe gemeint: Kinder oder Jugendliche, Heiratsfähige, ,Senioren“ und so weiter. Also, wenn ich alles haben möchte, was über Kinder ge- 
sagt wird, z.B. Spiele, Lieder, dann muß es in dieser Zeile K kodiert sein und abgerufen werden können.

Messerli: Ein Kinderlied ist also H9, weil Lied, und auf dem Index K ist als Altersgruppe ,Kind“ kodiert?

Levin: Genau. Aber es gibt noch andere Dinge, die das Soziale betreffen. Zum Beispiel Wohnort, etwa Städter oder Dorfbewohner. Gruppenspezifisch sind auch Berufe. Eine weitere Gruppe ist für den Glauben vorgesehen, eine andere für ,Banden', für Diebe, für ,Ganoven'. Auch diese bilden eine ,Innung 'im Orient, sie sind wohl auch schon in Deutschland organisiert. Auch Killer ist ein Beruf, wie etwa der Richterstand. Also, ich kann eine ganze Menge von Inhalten im sozialen Bereich kombinieren, und zwar auf einer bestimmten Ebene kodieren und leicht per Synthese abrufen. Ich kann es auch als Kartogramm darstellen, somit auch korrelieren. Auch das ist volkskundlich wichtig.

Dann gibt es eine Koordinate für ,Zeitliches ‘; das ist die zweite Qualifikation, Index L. Es gibt ja Dinge, die zu einer bestimmten Zeit stattfinden, innerhalb eines Wochen- oder Tageszyklus. Aber es gibt auch den Lebenslauf und Jahreslauf. Es sind alles Zeitumstände. Hier gibt es zum Beispiel etwas, das einer Person geschehen ist, als diese Soldat war oder im Gefängnis saß. Das ist auch eine ,Zeit'. Und dann: Wie wird überhaupt umgangssprachlich ,Zeit' markiert? Vor oder nach dem Krieg, vor oder nach der Flut, vor oder nach der Vertreibung, nach einer Katastrophe, etwa Feuerschaden, nach verschiedenen auffälligen Ereignissen im privaten Leben oder in der Gemeinschaft. All das hat mit „Zeit‘ zu tun, wovon sehr viel erzählt und aufgeschrieben wird.

Nun haben wir die soziale Schichtung in der siebten Gruppe, K, sowie die Zeitumstände in der achten Gruppe, L, ein wenig erörtert. Man braucht aber auch zu wissen, wie etwas gemacht und gebraucht wird. Darüber informiert der Kodifikator in der neunten Gruppe, Index M. Wenn Sie das Essen beschreiben, stellt sich die Frage: Wie wird die Speise zubereitet? Es kann gebraten, auch gekocht oder geräuchert sein. Man sollte auch die Geschmacksrichtung fixieren: Z.B. wird die gleiche Speise in gewissen Gegenden salzig, in anderen lieber süß gegessen. Die Grenze hat eine kulturhistorische Relevanz. Da brauchen Sie nur eine Ziffer aus einer kurzen Liste, dann haben Sie das, was nötig ist. Und: Woraus wird eine Speise gemacht? Da der Mensch nicht alles ißt, stellt sich die Frage, ob es Fleisch, Fisch, Milch oder Getreide ist. Damit kann ich schon eine Speise beschreiben. Ich kann sagen, ob sie gebraten, gebacken oder gerieben wurde. Das geht ganz einfach, ist aber volkskundlich sehr wichtig. Ebenso gehe ich vor, wenn es sich um Stickmuster oder andere Handarbeitsmuster handelt. Mit einem kurzen Kode Index M und drei Ziffern kann ich angeben, um welche Arten von Mustern es sich handelt, womit sich Museologen plagen. Man kann die Formen, auch etwa die des Gebäcks, leicht kodieren.

Eine andere sehr beliebte Wie-Frage, worüber Folkloristen schreiben, ist: Wie wird es erzählt? Zum Beispiel, mit Einschiebseln von Liedern oder ohne Lieder. Ein Epos kann von Afrikanern erzählt oder auch nur getanzt werden. Solche Dinge sind im Kodifikator vorgesehen. Bei den Liedern kommt es auf den Rhythmus, das Tempo an, auf die Art und Weise, wie ein Lied gesungen wird. Das alles kann 
man in ein bestimmtes Raster packen. Und jeder kann diese Fragen beantworten, auch wenn er keine Schulung gehabt hat. Das ist ganz einfach, wie Matti Kuusi auf deutsch zu sagen pflegte: ,idiotensicher'.

Messerli: Wenn Ihre Mitarbeiter einen Text, nachdem sie ihn vom Tonband transkribiert oder dem Archiv entnommen haben, nach Ihren Kategorien kodierten, hat das dann noch jemand kontrolliert?

Levin: Ja, möglichst gründlich. Also, da sitzen zwei, drei, vier Mitarbeiter und bearbeiten das. Sie haben einen Text und machen sich Notizen, indem sie jede Reihe abarbeiten, und am Ende bekommen sie im Konsens eine ,Signatur'. Die Signatur besteht aus sechs Klassifikationen und aus vier Qualifikationen. Bei den Qualifikationen können Sie viele Charakteristika zusammen nennen und jedesmal sind das nur der Index A, B, C, D, F, H, K, L, M nebst drei Ziffern, nicht mehr. $\mathrm{Ja}$, und noch eine Sparte kommt hinzu: die zehnte Gruppe mit Index Z. Das sind technische Angaben, zum Beispiel: Handelt es sich um ein Manuskript, oder ist es etwas Gedrucktes, ist es mit Tinte geschrieben oder mit Bleistift? Wenn es eine Tonaufnahme oder ein Video ist, gibt es auch Parameter für die Tonträger, für die Geräte. Und dann ist auch für Museen und Archive die Frage wichtig: In welchem Zustand ist das? Wenn es heißt: in einem schlechten Zustand, dann muß man kopieren und konservieren. Und dann kommen auch die Sprachen. Es kommen ja wirklich nicht so viele Sprachen und Mundarten in Betracht. Man notiere nicht den Namen der Sprache, sondern die Gruppe. Und wenn Sie alle Belege für die zehn Sparten durchgearbeitet haben, zwar nur auf einer bestimmten Ebene, dann haben Sie etwas geleistet und gelernt. Sie haben das Rohmaterial registriert, aufgenommen, ja dokumentiert.

Danach folgt die nächste Stufe: die Forschungsarbeit auf zweiter, höherer Ebene. Gesetzt den Fall, es handelt sich um Vierzeiler, und jetzt haben Sie Lust bekommen, sämtliche Vierzeiler zu untersuchen. Rufen Sie zuerst mal die Adressen Index von A ab, um die einzelnen Vierzeiler zu finden. Nachdem Sie nun sämtliche registrierte Vierzeiler gefunden haben, muß man die Texte, auch wenn es sich um Hunderttausende von Belegen handelt, alle samt Signatur auf Kärtchen kopieren. Das nächste Ziel ist, dafür zu sorgen, daß die Varianten innerhalb der Sammlung zusammenkommen.

Messerli: Wie macht man das?

Levin: Ein Vierzeiler hat etwa 25 Wörter. Nun tun Sie folgendes: Unterstreichen Sie mit schwarzem Bleistift die Sinnwörter, etwa die Substantive (also nicht die Bindewörter). Und dann kopieren Sie den Vierzeiler so viele Male, wie Sie Kennwörter unterstrichen haben. Nun bekommen Sie identische Karten von Texten samt Signaturen. Jetzt nehmen Sie einen Rotstift, unterstreichen damit auf der ersten Kopie das erste schwarz unterstrichene Wort rot, und dann das zweite unterstrichene Wort auf dem nächsten Kärtchen und so weiter. Danach ordnen Sie bitte die rot unterstrichenen Kärtchen bzw. die rot unterstrichenen Wörter der ganzen Sammlung nach dem ABC. Meine Tadschiken waren ganz verwundert: Ähnliche Texte haben sich mechanisch eingefunden in einer Kiste, an einer Stelle. Gliedern Sie jetzt die ,Typen ‘ ähnlichen Inhalts aus, so wird es möglich, die Texte als Varianten zu erfassen. Für jeden Typ läßt sich nun eine Synopse der Varianz 
kompilieren und auf Repertoireebene auswerten. Auch schon die mittlere Ebene eines Typs läßt sich monographisch hervorragend bearbeiten und nebenbei lassen sich Anthologien oder gar Korpuswerke herausgeben. Dieses Vorgehen würde übrigens das allenthalben gesunkene Niveau der Folkloristik sehr heben.

Wir hatten so die Möglichkeit, nicht nur mit Vierzeilern, sondern auch mit Sprichwörtern, Witzen und auch mit Tierfabeln zu ganz neuen Einsichten zu kommen. Als Nebenprodukt sind Kennwortschätze für Textsorten oder Gattungen (NB! auf Repertoireebene) mit Angabe der Frequenz, der Häufigkeit, entstanden. Hier ist es geboten, unsere Errungenschaften und methodischen Verfahren von den eiligen Autoren der ,Häufigkeitswörterbücher' für ganze Sprachen abzusetzen, die vermöge von ,Häufigkeit' Völker geistig charakterisieren wollen. Mit Unterstützung von Computern hat man im Sog der amerikanischen Linguistik eine ganze Reihe von Häufigkeitswörterbüchern als Dissertationen geschaffen und vermarktet. Man hat versucht, mathematisch, statistisch an die Frequenz von Einzelwörtern heranzugehen. Wirkliche Erkenntnisse wurden damit auch für die Literaturwissenschaft nicht gewonnen. Was ist der Aussagewert für einen Text, wenn man weiß, wievielmal ein Wort vorkommt? Z.B. sind die Wörter Pferd, Gaul, Roß, Mähre, Stute, Hengst zwar sachlich verwandt, aber sie zeigen verschiedene Häufigkeiten. Was kann man daraus folgern? So gut wie nichts. Aber wenn man es als Sachgruppe betrachtet und mit einer anderen Sachgruppe, z.B. ,wilde Tiere oder Haustiere', innerhalb einer ,Gattung' oder eines Zeitalters vergleicht, läßt sich schon vieles sagen. Aussagekräftig für eine Sprach- bzw. Literaturwissenschaft, ja für eine Gemeinschaft ist nicht die Frequenz einzelner Wörter, sondern die der inhaltlichen Sachgruppen. Der Versuch, in Wörtern gleicher Häufigkeit signifikante gemeinsame inhaltliche Eigenschaften zu entdecken, war nicht ergiebig, zumal man bemüht war, das Wesen einer Sprachgemeinschaft, einer Zeitstufe und dergleichen dadurch zu charakterisieren. Aufschlußreich war hingegen, die Bildung inhaltlicher Sachgruppen und die Summa von deren Frequenz vergleichend zu interpretieren. Kurz: Man muß zuerst einen Wortschatz inhaltlich gliedern, sei es als Wortfelder oder Sinnbezirke, ja, schlicht nach Sachgruppen. Ich habe dies am Lexikon der tadschikischen und armenischen Vierzeiler angewandt. Ich habe die Ergebnisse meinen Mitarbeitern als Dissertationen angedeihen lassen. Daraus konnte man sehr viel lernen. Das heißt in deutschen Landen Sprachinhaltsforschung (vgl. Jost Trier, Leo Weisgerber und Franz Dornseiff), und das ist es, was ich Ihnen beweisen kann und gern als ,Überbau' zur Volkskunde, als Dach der Folkloristik verständlich machen möchte.

Mir war es wichtig, einmal den behaupteten Unterschied von Gattungen anhand von Korpuswerken zu überprüfen. Worin unterscheiden sich inhaltlich Vierzeiler von Zweizeilern und/oder Sprichwörtern? Noch wichtiger war zu klären: Worin unterscheiden sich (wenn überhaupt) auf Korpusebene tadschikische (,islamische") Vierzeiler von armenischen (,christlich-monophysitischen'), die zu gleicher Zeit und unter ähnlichen Umständen gesammelt wurden? Gibt es eine nationale (ethnische) Spezifik, wie es in der UdSSR anfangs vulgärmarxistisch verneint, aber seit den dreißiger Jahren gepredigt wurde? Solch eine kritische Fragestellung und eine gebührende Grundlage von repräsentativen Belegen bleiben 
in der Folkloristik einmalig. Ich bin nicht geneigt, meine Methoden und Forschungsverfahren patentieren zu lassen, ich habe sie vielmehr teilweise durch das Wissenschaftskolleg zu Berlin freigestellt. Allerdings war es unangenehm festzustellen, daß ein druckfertiges Korpuswerk von Schwänken und Witzen, das ich im Auftrag des Instituts für Sprache und Literatur in Duschanbé dem Orientalistischen Verlag in Moskau zur Veröffentlichung brachte, von meinem Mitarbeiter einfach beim Umzug aus dem Verlag gestohlen und als eigenständige Dissertation vorgelegt wurde. Freilich, so etwas war keine Seltenheit. Man rechnete damit und befürchtete, daß ich emigriere, was erfahrungsgemäß für ein sowjetisches Institut wie ein Schlag wirkte. Man war bemüht, solch ein Glied zu extrahieren, womöglich erst zu diffamieren. Das in Moskau beim Verlag für Orientalistik herausgegebene Korpuswerk tadschikischer Fabeln als Ergebnis kollektiver Arbeit unter meiner Leitung wurde meines Wissens nicht rezipiert, obwohl es zukunftweisend konzipiert wurde. Auch eine Reihe von Dissertationen meiner Mitarbeiter, die ich projektiert und von Anfang bis zum Ende geleitet habe, sind in Gestalt von Autoreferaten, welche von mir in russischer Sprache formuliert wurden, im Druck erschienen. Sie wurden im Untertitel als ,Versuch einer quantitativen Analyse“ präsentiert. Das war ein Novum.

Messerli: Haben Sie noch Zugang zu Ihren ehemaligen Mitarbeitern und zum Archiv, wenn Sie etwas benötigen?

Levin: Das ist das Tragische - für sie mehr, als für mich. Nein, ich habe keinen Zugang mehr. Meine Lebensregel lautete: „Omnia mea mecum porto.“ Denn Tadschikistan und Armenien sind Ausland geworden. Tadschikistan befindet sich, wie das Nachbarland Afghanistan, wo viele Tadschiken wohnen, im Bürgerkrieg. Gesetzt den Fall, ich möchte dorthin fahren, um etwas nachzuschlagen im Archiv, so wäre dies kaum möglich. Amerikaner und Afghanen kommen manchmal nach Duschanbé und bewundern dort die Ordnung. Folgendes ist geschehen: Ein Mann aus dem Iran ist gekommen und hat gefragt: „Haben Sie Vierzeiler? Ich habe da einen Vierzeiler; haben Sie vielleicht Varianten dazu?" Er meinte, es würde Wochen dauern, bis man das herausfände. Ein Mitarbeiter ging hin, und nach zwei, drei Minuten hat er ihm die Varianten, die ganze Gruppe zu diesem Text, gebracht, allerdings in russischer, weder arabischer noch lateinischer Schrift. In Tadschikistan habe ich sehr viel investiert, an Energie, an Wissen, an Geduld. Jetzt ist dort alles isoliert und vergammelt. Gesetzt den Fall, ich würde nach Tadschikistan gehen, würde man mich wahrscheinlich töten, wie es mit dem Präsidenten der Akademie geschah. Ja, weil ich aus dem Westen komme und sie modernisieren wollte, und noch verschiedene andere Motive kommen hinzu. Es genügt, den Artikel Tadschiken in der Enzyklopädie des Märchens zu lesen, um die dortige Lage zu begreifen.

In Armenien ist die Situation eine andere. Die Armenier haben genug gelitten und gewannen dadurch eine ganz andere Einstellung. Mit Armeniern habe ich noch Kontakte. Sie schreiben mir oder telefonieren, gratulieren mir zum Geburtstag. 